\title{
Berberine ameliorates severe acute pancreatitis-induced intestinal barrier dysfunction via a myosin light chain phosphorylation-dependent pathway
}

\author{
HONG-YIN LIANG* ${ }^{*}$ TAO CHEN* ${ }^{*}$ HONG-TAO YAN* ${ }^{*}$ ZHU HUANG and LI-JUN TANG \\ PLA Center of General Surgery, General Hospital of Chengdu Military Command, Chengdu, Sichuan 610083, P.R. China
}

Received August 8, 2013; Accepted February 21, 2014

DOI: $10.3892 / \mathrm{mmr} .2014 .1996$

\begin{abstract}
Berberine is a traditional drug used to treat gastrointestinal disorders in China and has been demonstrated to attenuate intestinal barrier dysfunction in certain animal models. However, the effects of berberine on pancreatitis-induced intestinal barrier dysfunction are yet to be fully elucidated. This study aimed to investigate the effect of berberine pretreatment on the attenuation of intestinal barrier dysfunction induced by severe acute pancreatitis (SAP). A total of 36 rats were randomly divided into Sham, SAP and SAP plus berberine groups. Pancreatitis was induced using retrograde injection of $3 \%$ Na-taurocholate into the pancreatic duct. Histological examinations of the pancreas were performed and intestinal barrier dysfunction was characterized by histological measurements and the assessment of serum diamine oxidase activity and endotoxin levels. Zonula occludens-1 and occludin mRNA and protein expression, as well as myosin light chain (MLC) phosphorylation, were assessed. SAP rat models were successfully established. Berberine treatment was found to have no significant effect on the histological changes in the pancreas, but was observed to ameliorate the intestinal mucosal barrier damage and membrane permeability associated with SAP. Although berberine exerted minimal effects on tight junction proteins in the ilea of SAP rats, it was observed to significantly inhibit SAP-induced MLC phosphorylation. To the best of our knowledge, this is the first study to demonstrate that berberine attenuates SAP-induced intestinal barrier dysfunction in vivo. In addition, this study shows that the effect of berberine on intestinal barrier function may be associated
\end{abstract}

Correspondence to: Dr Li-Jun Tang, PLA Center of General Surgery, General Hospital of Chengdu Military Command, 270 Rongdu Street, Chengdu, Sichuan 610083, P.R. China

E-mail: tanglijuncd@126.com

*Contributed equally

Key words: berberine, acute pancreatitis, intestinal barrier function, rat model with the inhibition of SAP-induced upregulation of MLC phosphorylation.

\section{Introduction}

Severe acute pancreatitis (SAP) is a systemic disease primarily characterized by pancreatic self-necrosis. SAP involves a complex array of mediators that are capable of initiating and exacerbating systemic inflammatory response syndrome and, in severe cases, multiple organ dysfunction syndrome (MODS). Despite improvements in treatment, SAP remains associated with a mortality rate of between 15 and $30 \%(1,2)$. Experimental studies have shown that the intestine is one of the target organs vulnerable to injury and the development of MODS $(3,4)$. The intestinal mucosal barrier plays an important role in maintaining intestinal function by preventing bacteria and toxins in the enteric cavity from passing into the bloodstream. The infection complications associated with SAP may be a result of bacterial translocation from the gastrointestinal tract due to increased intestinal permeability $(5,6)$.

Berberine is the major constituent of the Coptidis rhizome, which has been widely used as a traditional drug for the treatment of gastrointestinal disorders in China. Studies have revealed that berberine has pleiotropic biochemical and pharmacological effects, including anti-inflammatory, anti-bacterial, anti-apoptotic and anti-tumor actions (7-9). Of note, berberine has been demonstrated to attenuate intestinal barrier dysfunction in certain animal models. For example, berberine has been found to ameliorate intestinal mucosal barrier damage in trinitrobenzene-sulfonic acid (TNBS)- and dextran sulfate sodium-induced experimental colitis in mice $(10,11)$. Furthermore, berberine has been shown to protect against lipopolysaccharide- or pro-inflammatory cytokine-induced intestinal barrier injury in mice, through the regulation of tight junctions (TJs) and the myosin light chain kinase (MLCK) pathway (12-14).

The effects of berberine on the intestinal mucosa in SAP-induced intestinal injury are yet to be fully elucidated. In the present study, it was hypothesized that berberine was likely to attenuate damage to the intestinal epithelial barrier in a rat model of SAP. This study aimed to investigate whether berberine, which protects the intestinal mucosal barrier, is capable of reducing intestinal permeability and bacterial translocation in rats with SAP. 


\section{Materials and methods}

Animals and experimental design. Healthy, adult Sprague Dawley rats weighing between 250 and $280 \mathrm{~g}$ were purchased from Dashuo Laboratories (Chengdu, China). All animals were individually housed in plastic cages containing wood shavings, and maintained in a temperature-controlled environment with a 12-h light/dark cycle and free access to food and water. All animals were allowed to acclimate to these conditions for 1 week prior to experimental treatment. All experimental animal procedures were approved by the Ethics Committee for Animal Experiments of the General Hospital of Chengdu Military Command (Chengdu, China), and all animal experiments were performed according to the National Animal Welfare Law of China.

Thirty-six Sprague Dawley rats were randomly divided into sham-operated, SAP and SAP plus berberine groups ( $n=12 /$ group), which were referred to as the Sham, SAP and SAP+ber groups, respectively. Berberine (Sigma-Aldrich, St. Louis, MO, USA; $50 \mathrm{mg} / \mathrm{kg}$ dissolved in $1 \mathrm{ml}$ normal saline/200 g body weight) or normal saline $(1 \mathrm{ml} / 200 \mathrm{~g}$ body weight) were administered intragastrically once a day for 5 days, prior to the induction of pancreatitis.

After $12 \mathrm{~h}$ of fasting, rats were anesthetized with an intraperitoneal injection of $50 \mathrm{mg} / \mathrm{kg}$ phenobarbital and a midline laparotomy was performed. In the Sham group, upon opening the abdominal cavity, the pancreas and duodenum were only moved prior to the closure of the abdominal wall, using a double layer of sutures, and the return of the rats to their cages. In the SAP and SAP+ber groups, subsequent to opening the abdominal cavity, pancreatitis was induced using retrograde injection of 3\% Na taurocholate (Sigma-Aldrich; $1 \mathrm{ml} / \mathrm{kg}$ body weight) into the pancreatic duct over a period of $2 \mathrm{~min}$. Following the surgery, rats in all three groups were injected subcutaneously with $5 \mathrm{ml} 0.9 \% \mathrm{NaCl}$ solution twice a day to supplement the blood volume. Twenty-four hours after the surgery, rats were re-anesthetized and laparotomies were performed. Samples of blood and tissue were obtained immediately.

Histological examination and pathological scoring. Ileal and pancreatic specimens were fixed in a $4 \%$ paraformaldehyde solution immediately following isolation. Tissues were then fixed, dehydrated, paraffin-embedded and sectioned, according to standard methods. The $4-\mu$ m-thick sections were stained with hematoxylin and eosin $(\mathrm{H} \& \mathrm{E})$, and the pathological changes in the small intestine and pancreas were examined using an optical microscope (Olympus, Tokyo, Japan). A pathologist who was blinded to the grouping scored the ileal specimens according to the method described by Chiu et al $(15,16)$. The mucosal damage was graded from zero (normal) to five (severely damaged).

Serum endotoxin and diamine oxidase (DAO) analysis. Serum DAO activity and endotoxin levels are used as indices of small intestinal mucosal mass and integrity. Rat blood samples were collected as described above, measured and then centrifuged at $1,509 \mathrm{x} \mathrm{g}$ for $10 \mathrm{~min}$ at $4^{\circ} \mathrm{C}$. The supernatant was transferred to sterile labeled tubes and stored at $-80^{\circ} \mathrm{C}$ until use. Serum endotoxin levels were assessed using a Chromogenic
Limulus Amebocyte Lysate assay kit (Shanghai Med \& Chem Institute, Shanghai, China). DAO activity was examined using a commercial kit (Nanjing Jianchen Co. Ltd., Nanjing, China) according to the manufacturer's instructions. In brief, DAO catalyzes the oxidation of the substrate putrescine, and the product is quantitatively oxidized by peroxidase in proportion to the quantity of hydrogen peroxide produced, resulting in the production of o-dianisidine, which has an absorption maximum at $440 \mathrm{~nm}$.

Bacterial culture of mesenteric lymph nodes (MLNs). MLNs were harvested under sterile conditions, prior to being homogenized, incubated at $37^{\circ} \mathrm{C}$ in an agitated water bath for $18 \mathrm{~h}$, plated on MacConkey II agar (Oxoid Ltd., Basingstoke, UK) and incubated aerobically at $37^{\circ} \mathrm{C}$ for $24 \mathrm{~h}$. A blinded visual inspection was then performed. Results were recorded as positive for growth, without quantification, or no growth. The culture was considered positive if bacterial growth was observed, and the incidence of bacterial translocation was calculated by determining the number of rats with a positive bacterial culture divided by the total number of rats studied.

Quantitative polymerase chain reaction ( $q P C R$ ) analysis. The rats were sacrificed by overdose of anesthesia and segments of the ileum were isolated, flushed and immediately placed in RNAiso Plus buffer (Takara Biotechnology Co. Ltd., Dalian, China). Total RNA was extracted according to the manufacturer's instructions. cDNA was synthesized by reverse transcription using the PrimeScript RT Reagent kit (Takara Biotechnology Co. Ltd.) with an oligo (dT) primer. qPCR analysis was performed using $\mathrm{SYBR}^{\circledR}$ Premix EX Taq ${ }^{\mathrm{TM}}$ II (Takara Biotechnology Co. Ltd.) in a Bio-Rad IQ5 system (Bio-Rad, Hercules, CA, USA). Primers for zonula occludens (ZO)-1, occludin and GAPDH were synthesized by Takara Biotechnology Co. and had the following sequences: ZO-1, 5'-GCTCCTCCCACCTCG CACGT-3' (forward) and 5'-GACCTGCTGGAGCATAGG GCTG-3' (reverse); occludin 5'-TGGAGTTGCGGGAGAGCG ATC-3' (forward) and 5'-GGGCAGTCGGGTTGACTCCCA-3' (reverse); GAPDH, 5'-TCCCTCAAGATTGTCAGCAA-3' (forward) and 5'-AGATCCACAACGGATACATT-3' (reverse). The reaction conditions were as follows: Initial denaturation at $95^{\circ} \mathrm{C}$ for $1 \mathrm{~min}$, template denaturation at $95^{\circ} \mathrm{C}$ for $20 \mathrm{sec}$, annealing at $60^{\circ} \mathrm{C}$ for $30 \mathrm{sec}$, extension at $72^{\circ} \mathrm{C}$ for $1 \mathrm{~min}$ (for a total of 40 cycles) and a final extension at $72^{\circ} \mathrm{C}$ for $10 \mathrm{~min}$. The cycle threshold (CT) was determined using automatic baseline calculations. A CT value of $>30$ was considered unacceptable. The relative gene expression was calculated using the $2^{-\Delta \Delta C \mathrm{CT}}$ method. GAPDH was used as an internal control.

Western blot analysis. Ilea were immediately placed in cold radio-immunoprecipitation assay lysis buffer (Beyotime Institute of Biotechnology, Haimen, China) subsequent to flushing. Each sample was then sonicated on ice three times for $30 \mathrm{sec}$ using a Sonic Dismembrator (Thermo Fisher Scientific, Waltham, MA USA) and centrifuged at 14,000 $\mathrm{x}$ g for $15 \mathrm{~min}$. Supernatants were then collected and the protein concentrations were determined according to the Bradford method using bicinchoninic acid assay reagent (Beyotime Institute of Biotechnology). Samples containing $20 \mu$ g protein were loaded 
Sham

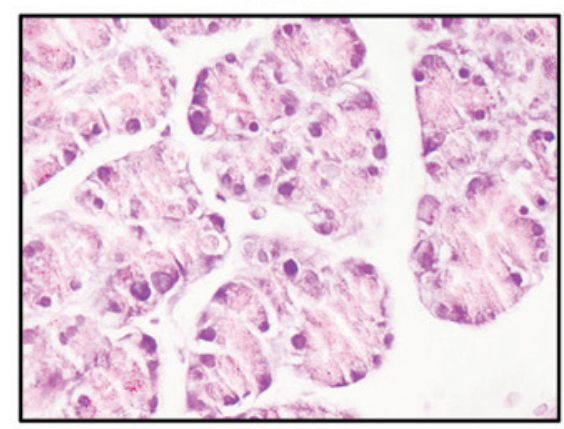

SAP

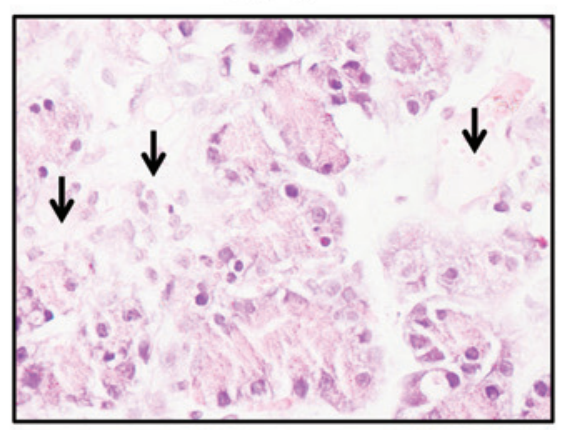

SAP+ber

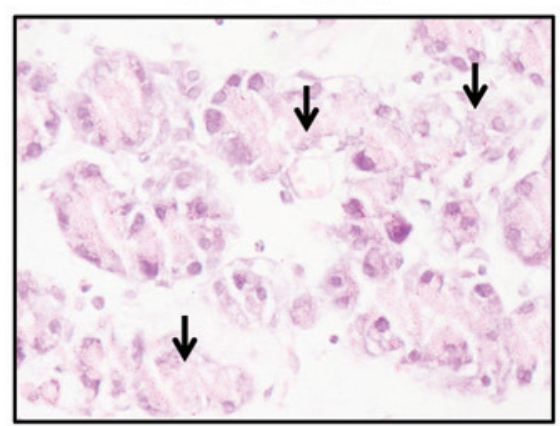

Figure 1. Pathological changes in the pancreas of rats in the Sham, SAP and SAP+ber groups. Representative micrographs of hematoxylin and eosin-stained pancreatic sections are shown. Acute pancreatitis was induced using retrograde injection of 3\% Na-taurocholate in the SAP and SAP+ber groups. Edema, acinar cell and adipocyte necrosis, monocyte and neutrophil infiltration and hemorrhage were observed in the pancreatic sections from the SAP and SAP+ber groups, while no injury was observed in those from the Sham group. Black arrows indicate pancreatic necrosis. Magnification, $\mathrm{x} 400$; $\mathrm{n}=12$ per group. SAP, severe acute pancreatitis; ber, berberine.

onto SDS-PAGE gels, electrophoresed using a Bio-Rad mini gel system (Bio-Rad) and then transferred to a polyvinylidene difluoride membrane (Millipore, Billerica, MA, USA). The membranes were blocked using $5 \%$ bovine serum albumin in $50 \mathrm{mM}$ Tris- $\mathrm{HCl}$ (pH 7.5), $140 \mathrm{mM} \mathrm{NaCl}$ and $0.1 \%$ Tween and incubated at $4{ }^{\circ} \mathrm{C}$ overnight with primary antibodies against ZO-1 (1:1,000; Santa Cruz Biotechnology, Inc., Santa Cruz, CA, USA), occludin (1:1,000; Santa Cruz Biotechnology, Inc.), MLC (1:1,000; Epitomics Inc., Burlingame, CA, USA) or phosphorylated MLC (pMLC; 1:1,000; Abcam PLC, Cambridge, MA, USA). Membranes were then washed three times and incubated with horseradish peroxidase-conjugated secondary antibodies. Membrane imaging was performed using an enhanced chemiluminesce detection system (Millipore) according to the manufacturer's instructions.

Statistical analysis. Kruskal-Wallis and Mann-Whitney U tests were performed for comparisons among groups. The incidence of gram-negative bacterial translocation to the MLNs was assessed using a $\chi^{2}$ test. A value of $\mathrm{P}<0.05$ was considered to indicate a statistically significant difference. Statistical analyses were performed using SPSS 18.0 statistical software (SPSS Inc., Chicago, IL, USA).

\section{Results}

Pancreatic pathology. To determine whether SAP rat models were successfully established, sections of pancreatic tissue were stained with H\&E and examined using light microscopy (Fig. 1). Pancreatic tissues from the Sham group exhibited a normal macroscopic and histological appearance. By contrast, the H\&E-stained sections from the SAP and SAP+ber groups revealed atypical pancreatic architectures, with marked interstitial edema, acinar cell necrosis, leukocyte infiltration and scattered hemorrhage. These findings suggest that SAP rat models were successfully established; however, berberine had no significant effect on the histological changes in the pancreas.

Evaluation of intestinal barrier function. To identify the role of berberine in the maintenance of intestinal barrier function, the intestinal membrane permeability was evaluated by assaying serum DAO activity and endotoxin concentration (Fig. 2A and B). In the Sham group, the baseline levels of serum DAO activity and endotoxin concentration $24 \mathrm{~h}$ after the sham surgery were $0.235 \pm 0.053 \mathrm{kU} / 1$ and $0.218 \pm 0.086 \mathrm{EU} / \mathrm{ml}$, respectively. Serum DAO activity and endotoxin levels were observed to be higher in the SAP group than those in the Sham group $(\mathrm{P}<0.05)$. Furthermore, berberine pretreatment was observed to significantly decrease serum DAO activity $(33.0 \%)$ and endotoxin levels $(16.7 \%)$ compared with levels in the SAP group $(\mathrm{P}<0.05)$.

To further assess the effect of berberine on intestinal barrier function, the incidence of bacterial translocation was evaluated (Fig. 2C). No bacterial translocation was observed in the MLNs from the Sham group; however, the incidence of bacterial translocation to the MLNs in the SAP group $24 \mathrm{~h}$ after the retrograde injection of $3 \% \mathrm{Na}$-taurocholate was observed to be $83.3 \%$. Berberine treatment was found to reduce the rate of bacterial translocation to $58.3 \%$, which was significantly lower than that observed in the SAP group $(\mathrm{P}<0.05)$. Serum DAO activity and endotoxin levels were also observed to decrease with berberine treatment.

These results indicate that berberine treatment may ameliorate the intestinal mucosal barrier damage associated with SAP induced by the retrograde injection of Na-taurocholate.

Pathological scoring of the intestine. Animals were sacrificed $24 \mathrm{~h}$ after surgery. H\&E-stained ileal sections were examined using an optical microscope and scored by a pathologist who was blinded to the grouping, according to the aforementioned method. The results of the pathological scoring are shown in Fig. 3. In the Sham group, the epithelial cells were observed to be closely arranged in a regular fashion. Twenty-four hours after the induction of SAP, the ileal mucosa was injured. Vacuolated epithelial cells, shortened villi and infiltrating lymphocytes were observed. The mean pathological score of the ilea in the SAP group increased to 1.833 , compared with 0.333 in the Sham group $(\mathrm{P}<0.05)$. Compared with the rats in the SAP group, berberine administration was observed to have a protective effect against ileal mucosal injury, and the ilea of 
A

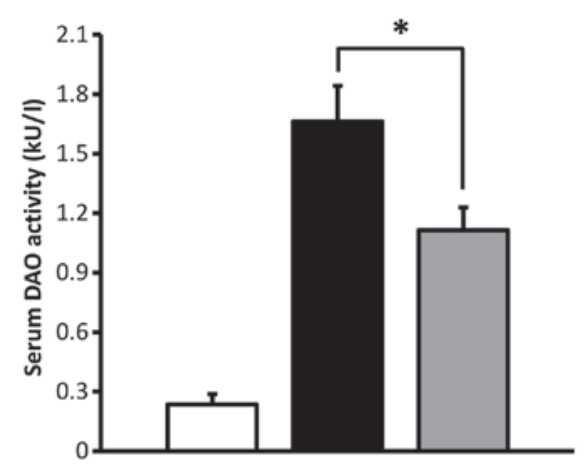

$\mathbf{C}$

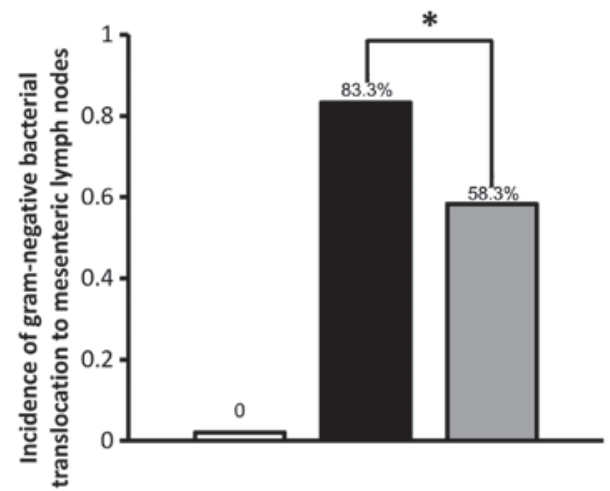

B

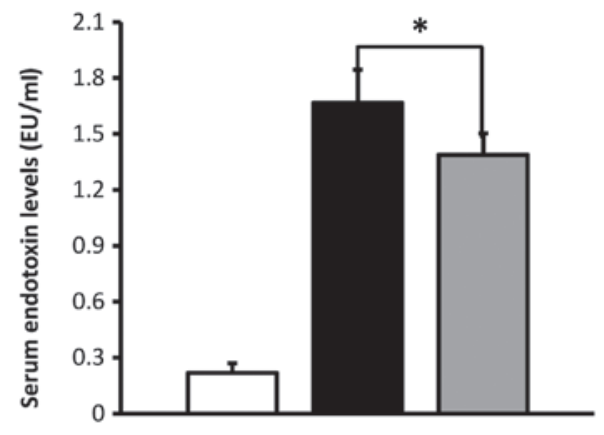

Figure 2. Evaluation of intestinal barrier function. Measurements of (A) serum DAO activity, (B) serum endotoxin levels and (C) the incidence of bacterial translocation were performed to assess the effect of berberine on intestinal membrane permeability and barrier function. Berberine decreased the serum DAO activity and endotoxin levels and the incidence of bacterial translocation, all of which were increased with the SAP-induced deterioration of intestinal barrier function, to similar degrees. ${ }^{*} \mathrm{P}<0.05, \mathrm{n}=12$ per group. $\mathrm{SAP}$, severe acute pancreatitis; DAO, diamine oxidase.

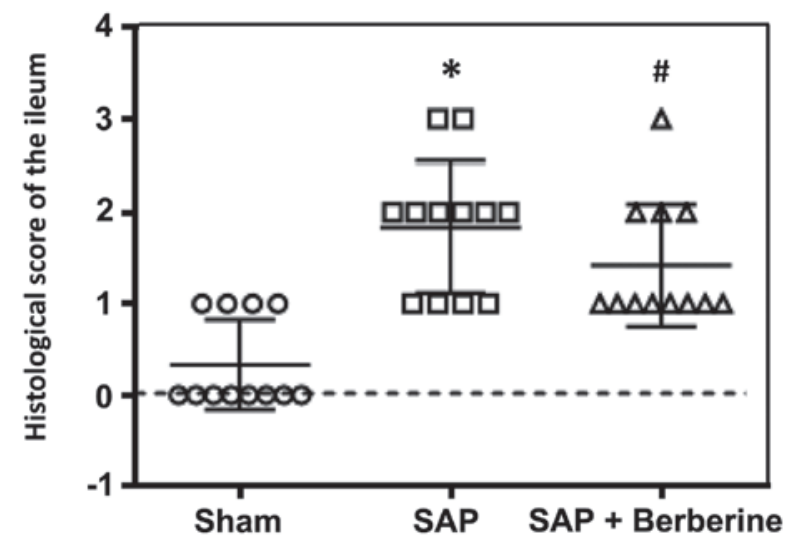

Figure 3. Histological scoring of the ileum. The pathological changes in the ileum were examined using an optical microscope and scored by a pathologist. Berberine was observed to ameliorate SAP-induced ileal damage. Data are presented as the mean \pm standard deviation. ${ }^{*} \mathrm{P}<0.05$ vs. the Sham group; ${ }^{\text {"}} \mathrm{P}<0.05$ vs. the SAP group. SAP, severe acute pancreatitis.

rats in the SAP+ber group exhibited relatively normal villi and mucosal integrity. The pathological scoring also demonstrated that berberine treatment attenuated the pancreatitis-induced mucosal injury compared with that observed in the SAP group (1.417 vs. 1.833 for the SAP+ber and SAP groups, respectively; $\mathrm{P}<0.05)$.

TJ alteration and MLC phosphorylation. TJs have a critical role in maintaining the function of the intestinal barrier $(17,18)$. The impairment of intestinal barrier func- tion is directly associated with the aberrant expression of TJ proteins (19). qPCR and western blot analyses were performed to detect the expression of the TJ proteins $\mathrm{ZO}-1$ and occludin. Berberine was not observed to influence ZO-1 and occludin mRNA expression in the SAP-induced rats (Fig. 4A). Furthermore, occludin protein expression did not differ significantly among the three groups; however, ZO-1 protein expression was observed to be significantly higher in the SAP+ber group than that in the SAP group (Fig. 4B and C). Overall, these results suggest that berberine exerts few effects on TJ proteins in the ilea of SAP rats.

It is well established that MLCK-mediated phosphorylation of MLC has a significant role in the physiological and pathophysiological regulation of intestinal epithelial TJs and paracellular leak pathways $(20,21)$. In the present study, although berberine was observed to have little effect on ZO-1 and occludin mRNA and protein expression, berberine was hypothesized to ameliorate SAP-induced barrier dysfunction through MLC phosphorylation, which, to the best of our knowledge, has not been previously investigated. As shown in Fig. 5, western blot analysis of MLC and pMLC revealed that berberine treatment had no significant effect on total MLC expression in SAP-induced rats. However, significantly higher pMLC levels were observed in rats in the SAP group compared with those in the Sham group (6.175-fold increase, $\mathrm{P}<0.05)$. This increase was significantly attenuated with berberine treatment, with pMLC levels in the SAP+ber group reduced to 0.349 -fold those in the SAP group $(\mathrm{P}<0.05)$. These data suggest that the inhibition of SAP-induced MLC phosphorylation may be one of the mechanisms responsible for 
A

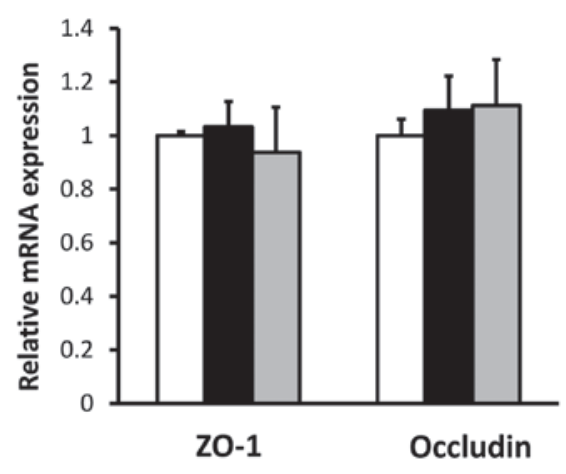

C
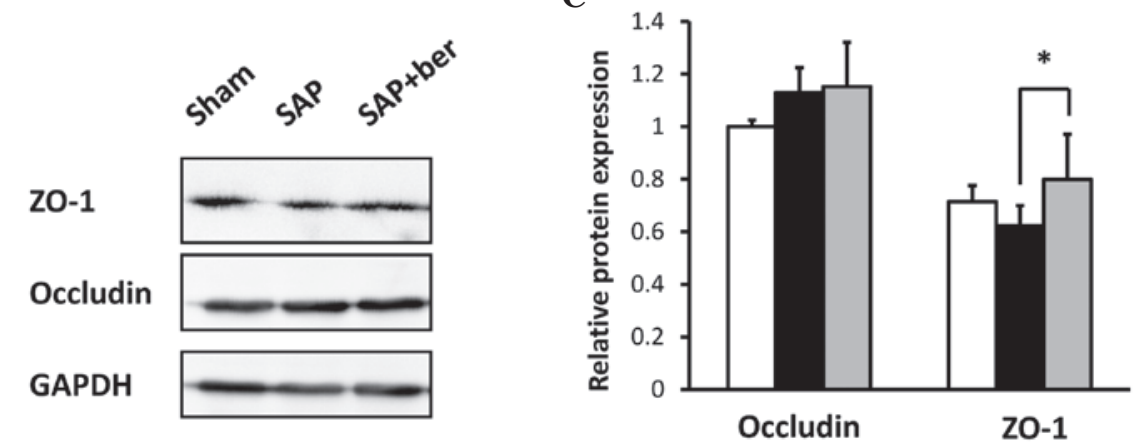

Figure 4. Alterations in the mRNA and protein expression of the tight junction components ZO-1 and occludin in SAP rats following berberine administration. (A) mRNA expression of ZO-1 and occludin. (B and C) Protein expression of ZO-1 and occludin. ${ }^{*} \mathrm{P}<0.05$ vs. the SAP group. SAP, severe acute pancreatitis; ber, berberine; ZO-1, zonula occludens.

A

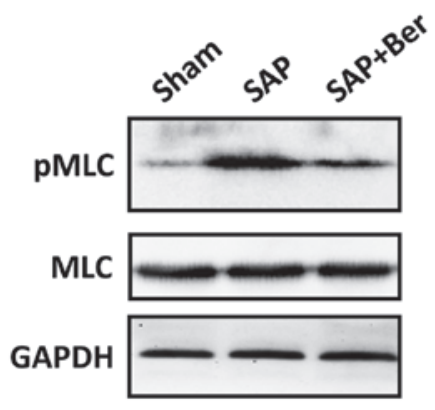

B

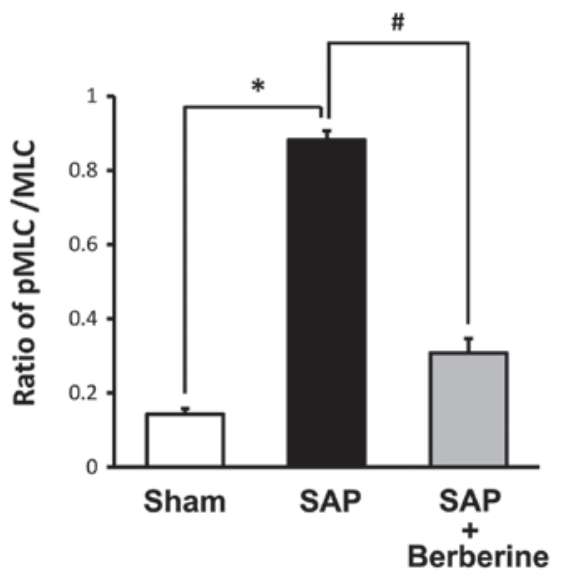

Figure 5. Effect of berberine on MLC phosphorylation in SAP rats. Berberine significantly suppressed the SAP-induced increase in pMLC in the ileum. ${ }^{*} \mathrm{P}<0.05$ vs. the Sham group; ${ }^{*} \mathrm{P}<0.05$ vs. the SAP group. SAP, severe acute pancreatitis; ber, berberine; MLC, myosin light chain; pMLC, phosphorylated MLC.

the beneficial effect of berberine on intestinal barrier function during SAP-induced damage.

\section{Discussion}

The present study investigated the effect of berberine on intestinal epithelial structure and barrier function in a rat model of SAP. Berberine was found to significantly prevent the loss of epithelial barrier function induced by pancreatitis in rat models of SAP, as evidenced by the reduction in permeability and bacterial translocation upon berberine administration. Furthermore, berberine was observed to inhibit the SAP-induced upregulation of pMLC levels, while the TJ proteins ZO-1 and occludin remained unaffected.
Although the etiology and pathogenesis of pancreatitis-induced intestinal barrier dysfunction are yet to be fully elucidated, it is well established that this dysfunction is characterized by the overproduction of various pro-inflammatory cytokines within the mucosa and the disruption of epithelial barrier function. SAP development is associated with the premature activation of pancreatic enzymes in the acini and an excessive inflammatory response, stimulating a cascade reaction $(22,23)$. The subsequent microcirculation disturbance-induced ischemia, hypoxia and ischemia-reperfusion injury have important roles in the development of extrapancreatic organ injury in $\operatorname{SAP}(24,25)$. The intestinal mucosal barrier plays a significant role in maintaining intestinal function and preventing the transfer of bacteria and toxins from the enteric 
cavity into the blood circulation. The infection complications caused by SAP may be a result of bacterial translocation from the gastrointestinal tract, and are associated with treatment failure $(26,27)$.

Berberine has been used as a remedy for gastrointestinal diseases, particularly diarrhea, for centuries. Recent studies have revealed that berberine has pleiotropic biochemical and pharmacological effects, including anti-inflammatory, anti-bacterial, anti-apoptotic and anti-tumor actions $(8,9)$. In addition, berberine has been demonstrated to attenuate intestinal barrier dysfunction in certain animal models. Lee et al (11) reported that berberine may improve TNBS-induced colitis by suppressing interleukin-8 expression. Furthermore, berberine has been shown to exhibit a protective effect against epithelial and endothelial barrier function in vitro $(28,29)$.

The effect of berberine on the intestinal mucosa in SAP-induced intestinal injury is yet to be fully elucidated. Therefore, the present study aimed to assess the effect of berberine on intestinal membrane permeability and bacterial translocation by measuring serum DAO activity, endotoxin levels and the incidence of bacterial translocation to the MLNs, all of which are techniques that have been widely used to assess intestinal barrier function. The results of this study demonstrate that berberine is capable of preventing the intestinal barrier dysfunction caused by SAP in vivo. However, the mortality rate, which may be valuable for the evaluation of the effect of berberine on pancreatitis, was not recorded due to the small sample size.

Although the molecular mechanism by which berberine attenuates SAP-induced intestinal barrier dysfunction is yet to be elucidated, a number of reports have provided novel insights into potential signaling pathways. Several investigations have shown that berberine attenuates pro-inflammatory cytokine-induced intestinal barrier dysfunction by ameliorating the effects on TJs in vitro and in vivo $(14,29,30)$. The opening of TJs is primarily dependent on the composition and organization of TJ proteins, particularly occludin, ZO-1 and claudins, which are responsible for barrier function. Occludin is a major TJ component which, upon phosphorylation, becomes redistributed, resulting in a significant decrease in transepithelial electrical resistance, indicating the occurrence of intestinal barrier injury (31). In a glioma cell line, berberine has been reported to decrease the activation of protein kinase $\mathrm{C}-\alpha$, which catalyzes occludin phosphorylation, leading to cytoskeletal rearrangements (32). However, in the present study, berberine was observed to have little effect on the TJ proteins ZO-1 and occludin.

MLCK and nuclear factor $\kappa$-light-chain-enhancer of activated $\mathrm{B}$ cells $(\mathrm{NF}-\kappa \mathrm{B})$ have been reported to have central roles in the alteration of intestinal epithelial TJs. TJ dysregulation induced by MLCK activation has been found to cause apoptosis-mediated barrier loss and experimental colitis (33). Furthermore, additional studies have shown that MLCK-dependent TJ regulation has an important role in thermal injury-induced intestinal barrier dysfunction $(34,35)$. In addition, berberine has been observed to ameliorate intestinal epithelial TJ damage and to downregulate MLCK pathways in an endotoxinemia model (14). Therefore, the present study assessed the MLCK pathway in rat models of SAP. The results of this study demonstrate that berberine is capable of suppressing SAP-induced pMLC upregulation, which may represent the molecular mechanism responsible for the protective effect of berberine against SAP-induced intestinal epithelial barrier dysfunction.

In conclusion, the present study showed that berberine may attenuate the intestinal barrier dysfunction induced by SAP in vivo. To the best of our knowledge, this is the first study to demonstrate that berberine is capable of inhibiting the SAP-induced upregulation of MLC phosphorylation.

\section{References}

1. Morel DR, Frossard JL, Cikirikcioglu B, Tapponnier M and Pastor CM: Time course of lung injury in rat acute pancreatitis. Intensive Care Med 32: 1872-1880, 2006.

2. Lankisch PG and Lerch MM. Pharmacological prevention and treatment of acute pancreatitis: where are we now? Dig Dis 24: 148-159, 2006.

3. Zhang XP, Zhang J, Song QL and Chen HQ. Mechanism of acute pancreatitis complicated with injury of intestinal mucosa barrier. J Zhejiang Univ Sci B 8: 888-895, 2007.

4. Gurleyik E, Coskun O, Ustundag N and Ozturk E. Prostaglandin E1 maintains structural integrity of intestinal mucosa and prevents bacterial translocation during experimental obstructive jaundice J Invest Surg 19: 283-289, 2006.

5. Suzuki T. Regulation of intestinal epithelial permeability by tight junctions Cell Mol Life Sci 70: 631-659, 2013.

6. Camilleri M, Madsen K, Spiller R, Greenwood-Van Meerveld B and Verne GN. Intestinal barrier function in health and gastrointestinal disease. Neurogastroenterol Motil 24: 503-512, 2012.

7. Boberek JM, Stach J and Good L. Genetic evidence for inhibition of bacterial division protein FtsZ by berberine PLoS One 5: e13745, 2010.

8. Saha P, Bhattacharjee S, Sarkar A, Manna A, Majumder S and Chatterjee M. Berberine chloride mediates its anti-leishmanial activity via differential regulation of the mitogen activated protein kinase pathway in macrophages. PLoS One 6: e18467, 2011.

9. Wang L, Liu L, Shi Y, et al: Berberine induces caspase-independent cell death in colon tumor cells through activation of apoptosis-inducing factor. PLoS One 7: e36418, 2012.

10. Yan F, Wang L, Shi Y, et al: Berberine promotes recovery of colitis and inhibits inflammatory responses in colonic macrophages and epithelial cells in DSS-treated mice. Am J Physiol Gastrointest Liver Physiol 302: G504-G514, 2012.

11. Lee IA, Hyun YJ and KimDH. Berberine ameliorates TNBS-induced colitis by inhibiting lipid peroxidation, enterobacterial growth and NF- $\kappa B$ activation. Eur J Pharmacol 648: 162-170, 2010.

12. Feng AW, Gao W, Zhou GR, et al: Berberine ameliorates COX-2 expression in rat small intestinal mucosa partially through PPAR $\gamma$ pathway during acute endotoxemia. Int Immunopharmacol 12: 182-188, 2012.

13. Li HM, Wang YY, Wang HD, et al: Berberine protects against lipopolysaccharide-induced intestinal injury in mice via alpha 2 adrenoceptor-independent mechanisms. Acta Pharmacol Sin 32: 1364-1372, 2011.

14. Gu L, Li N, Gong J, Li Q, Zhu W and Li J. Berberine ameliorates intestinal epithelial tight-junction damage and down-regulates myosin light chain kinase pathways in a mouse model of endotoxinemia J Infect Dis 203: 1602-1612, 2011.

15. Chiu CJ, McArdle AH, Brown R, Scott HJ and Gurd FN Intestinal mucosal lesion in low-flow states. I. A morphological, hemodynamic, and metabolic reappraisal. Arch Surg 101: 478-483, 1970

16. Chiu CJ, Scott HJ and Gurd FN. Intestinal mucosal lesion in low-flow states. II. The protective effect of intraluminal glucose as energy substrate. Arch Surg 101: 484-488, 1970.

17. Samak G, Suzuki T, Bhargava A and Rao RK. c-Jun NH2-terminal kinase-2 mediates osmotic stress-induced tight junction disruption in the intestinal epithelium. Am J Physiol Gastrointest Liver Physiol 299: G572-G584, 2010.

18. Strauman MC, Harper JM, Harrington SM, Boll EJ and Nataro JP. Enteroaggregative Escherichia coli disrupts epithelial cell tight junctions. Infect Immun 78: 4958-4964, 2010.

19. Anderson RC, Cookson AL, McNabb WC, et al: Lactobacillus plantarum MB452 enhances the function of the intestinal barrier by increasing the expression levels of genes involved in tight junction formation. BMC Microbiol 10: 316, 2010. 
20. Turner JR. Intestinal mucosal barrier function in health and disease. Nat Rev Immunol 9: 799-809, 2009.

21. Shen L, Weber CR, Raleigh DR, Yu D and Turner JR. Tight junction pore and leak pathways: a dynamic duo. Annu Rev Physiol 73: 283-309, 2011.

22. Kahl S and Mayer JM. Update on experimental acute pancreatitis. Minerva Gastroenterol Dietol 58: 355-363, 2012.

23. Lerch MM and Gorelick FS. Models of acute and chronic pancreatitis. Gastroenterology 144: 1180-1193, 2013.

24. Mayerle J, Dummer A, Sendler M, et al: Differential roles of inflammatory cells in pancreatitis. J Gastroenterol Hepatol 27 (Suppl 2): 47-51, 2012

25. Wan MH, Huang W, Latawiec D, et al: Review of experimental animal models of biliary acute pancreatitis and recent advances in basic research. HPB (Oxford) 14: 73-81, 2012

26. Jha RK, Yong MQ and Chen SH. The protective effect of resveratrol on the intestinal mucosal barrier in rats with severe acute pancreatitis. Med Sci Monit 14: BR14-BR19, 2008.

27. Wang X, Wang B, Wu J and Wang G. Beneficial effects of growth hormone on bacterial translocation during the course of acute necrotizing pancreatitis in rats. Pancreas 23: 148-156, 2001.

28. Amasheh M, Fromm A, Krug SM, et al: TNFalpha-induced and berberine-antagonized tight junction barrier impairment via tyrosine kinase, Akt and NFkappaB signaling. J Cell Sci 123: $4145-4155,2010$
29. Li N, Gu L, Qu L, et al: Berberine attenuates pro-inflammatory cytokine-induced tight junction disruption in an in vitro model of intestinal epithelial cells. Eur J Pharm Sci 40: 1-8, 2010.

30. Gu L, Li N, Li Q, et al: The effect of berberine in vitro on tight junctions in human Caco-2 intestinal epithelial cells. Fitoterapia 80: 241-248, 2009.

31. Marano CW, Garulacan LA, Ginanni N and Mullin JM. Phorbol ester treatment increases paracellular permeability across IEC-18 gastrointestinal epithelium in vitro. Digest Dis Sci 46: 1490-1499, 2001.

32. Lin TH, Kuo HC, Chou FP and Lu FJ. Berberine enhances inhibition of glioma tumor cell migration and invasiveness mediated by arsenic trioxide. BMC Cancer 8: 58, 2008.

33. Su L, Nalle SC, Shen L, et al: TNFR2 activates MLCK-dependent tight junction dysregulation to cause apoptosis-mediated barrier loss and experimental colitis. Gastroenterology 145: 407-415, 2013.

34. Guo M, Yuan SY, Frederich BJ, et al: Role of non-muscle myosin light chain kinase in neutrophil-mediated intestinal barrier dysfunction during thermal injury Shock 38: 436-443, 2012.

35. Zahs A, Bird MD, Ramirez L, Turner JR, Choudhry MA and Kovacs EJ. Inhibition of long myosin light-chain kinase activation alleviates intestinal damage after binge ethanol exposure and burn injury. Am J Physiol Gastrointest Liver Physiol 303: G705-G712, 2012 